\title{
Plain film diagnosis in meconium plug syndrome, meconium ileus and neonatal Hirschsprung's disease*
}

\section{A scoring system}

\author{
S.M.Hussain ${ }^{1}$, M.Meradji ${ }^{1}$, S. G.F.Robben ${ }^{1}$, and W.C.J.Hop ${ }^{2}$ \\ ${ }^{1}$ Department of Pediatric Radiology, Sophia Children's Hospital, Erasmus University, Rotterdam, the Netherlands \\ ${ }^{2}$ Department of Epidemiology and Biostatistics, Erasmus University, Rotterdam, the Netherlands
}

Received: 29 July 1991; accepted: 25 September 1991

\begin{abstract}
Abdominal plain films of 133 neonates, with 82 cases of meconium plug syndrome (MPS), 27 cases of meconium ileus (MI) and 24 cases of neonatal Hirschsprung's disease (HD), were reviewed to assess the value of such radiographs for diagnosis. The radiographs were examined according to a list of 11 parameters. By using multivariate discriminant analysis, it appeared that 4 parameters i.e. dilatation of bowel loops, varying loop calibre, fluid levels and colonic gas were most important in discriminating among the three disorders. For each parameter the weight (in points) was derived. To classify patients, three group-scores had to be calculated: the group-score with the largest value indicated the most likely disorder. So in $99 \%, 88 \%$ and $63 \%$ of MPS, HD and MI, respectively, an accurate diagnosis could be predicted. The overall diagnostic accuracy was $89 \%$. Such a diagnosis can be a sound basis for further investigation.
\end{abstract}

Meconium plug syndrome (MPS), meconium ileus (MI) and Hirschsprung's disease (HD) are functional disorders which cause evacuation problems of meconium in the neonate. MI is mostly related to cystic fibrosis (CF) [1]; however, cases without CF have been described $[2,3]$. $\mathrm{HD}$, congenital aganglionosis, most often mimics MPS, when it occurs in the neonate $[4,5]$.

The initial abdominal plain films in these disorders, have to be examined according to some defined criteria to make further diagnostic or therapeutic procedures more logical. Yet there is confusion and disagreement about these criteria $[1,4-8]$. Therefore, abdominal radiographs were reviewed to assess their value for diagnosis.

\section{Materials and methods}

This study included 133 neonates, admitted between 1976 and 1989 , with diagnoses of MPS ( 82 cases), MI (27 cases) and HD (24 cases).

\footnotetext{
* Presented at the 27th Annual Meeting of German Society of Pediatric Radiology, September 1990, Bern, Switzerland
}

Male:female ratio was 42:40, 17:10 and 14:10 respectively. The age at the time of the diagnosis ranged from 24 to 72 hours.

In each patient two abdominal plain films were available: a supine radiograph and a supine lateral view with horizontal beam. These radiographs were examined in a random order, without prior knowledge of the diagnoses, according to the following parameters:

- dilatation of bowel loops (DBL)

- varying loop calibre (VLC)

- fluid levels (FL)

- bowel wall edema (BWE)

- stretching of loops (SL)

- meconium mottling (MM)

- colonic gas (CG)

- rectal gas ( $\mathrm{RG})$

Other signs (perforation, ascites and calcification) were also searched for.

The diagnosis of MPS was accepted when symptoms relieved after a klysma or a Gastrografin enema and subsequently MI and $\mathrm{HD}$ were ruled out either by follow up or by means of a sweat test and a rectal biopsy.

The diagnosis of MI was established with the radiological evidence of small bowel obstruction caused by impacted meconium. Subsequently, in all operated cases the diagnoses of MI was confirmed. Ilial atresia, being a separate entity, was excluded. If necessary, the sweat tests were repeated during a follow up period of 9. 24 months.

The diagnosis of HD was only accepted with positive histochemistry and pathology of rectal biopsy material.

The findings were compared, using the $\chi^{2}$-test and Fisher's exact test with two-sided $P$-value considered significant at $P<0.05$.

In addition, multivariate discriminant analysis was used in weighting the various radiological parameters in relation to the classification of patients into the three diagnostic groups. The a-priori probabilities herein for each group were taken as the fractions of cases who belonged to the various groups i.e. MPS (82/133), MI (27/133) and HD (24/133).

\section{Results}

The outcomes of radiographs are shown in Table 1. Certain parameters were notable: in MPS, the dilatation of bowel loops occured without varying loop calibre or fluid levels; in MI, the dilatation of loops with varying loop 
Table 1. Plain film findings in meconium plug syndrome (MPS), meconium ileus (MI) and neonatal Hirschsprung's disease (HD). The three groups are compared to each other. NS $=$ not significantly different $(P>0.05)$

\begin{tabular}{|c|c|c|c|c|c|c|c|c|c|}
\hline & \multicolumn{2}{|c|}{$\begin{array}{l}\text { Meconium } \\
\text { plug syndrome } \\
\text { ( } 82 \text { cases) }\end{array}$} & \multicolumn{2}{|c|}{$\begin{array}{l}\text { Meconium } \\
\text { ileus } \\
(27 \text { cases })\end{array}$} & \multicolumn{2}{|c|}{$\begin{array}{l}\text { Hirschsprung's } \\
\text { disease } \\
\text { (24 cases) } \\
\end{array}$} & \multirow[t]{2}{*}{$\begin{array}{l}\text { MPS-MI } \\
(P \text {-values })\end{array}$} & \multirow[t]{2}{*}{$\begin{array}{l}\text { MPS - HD } \\
(P \text {-values })\end{array}$} & \multirow[t]{2}{*}{$\begin{array}{l}\mathrm{MI}-\mathrm{HD} \\
(P \text {-values })\end{array}$} \\
\hline & $(n)$ & {$[\%]$} & $(n)$ & {$[\%]$} & $(n)$ & {$[\%]$} & & & \\
\hline 2. Varying loop calibre (VLC) & 1 & 1 & 17 & 63 & 0 & - & $<0.001$ & NS & $<0.001$ \\
\hline 3. Fuid levels (FL) & 0 & - & 7 & 26 & 17 & 71 & $<0.001$ & $<0.001$ & $<0.001$ \\
\hline 4. Bowel wall edema (BWE) & 0 & - & 1 & 4 & 3 & 13 & NS & 0.01 & NS \\
\hline 7. Colonic gas (CG) & 21 & 26 & 2 & 7 & 19 & 79 & 0.06 & $<0.001$ & $<0.001$ \\
\hline 8. Rectal gas ( $\mathrm{RG}$ ) & 12 & 15 & 2 & 7 & 0 & - & NS & NS & NS \\
\hline
\end{tabular}

calibre, was characteristic; and in HD, the loops were dilated without variation in loop size, while the signs of fluid levels and colonic gas were frequent (Fig.1).

In addition, perforation was seen in MPS and HD (one case each). Ascites occured in MPS ( 1 case), in MI ( 3 cases) and in HD ( 2 cases). No calcification was noted.

Using discriminant analysis, it appeared that the four variables DBL, VLC, FL and CG (Table 1) were the most important ones in discriminating among the three groups. No additional value was found for other items.

To classify patients, three group-scores, in which the parameters were weighted, needed to be calculated. The weight (in points) of each parameter is given in Table 2. A patient had to be classified into the group which obtained the largest score. The points for a particular sign were given only in the presence of it, otherwise the score was zero points. For example, a patient with the presence of DBL, absence of VLC, presence of FL and presence of CG receives the three scores: MPS-score: $12+0+0+$ $1+3=16$, MI-score: $11+0+3+0+0=14$ and HD-score: $6+0+10+5+1=22$. As the HD-score is the largest of the three, HD is the most likely disease for this case.

In applying this derived scoring system, an accurate diagnosis could be predicted in $99 \%, 88 \%$ and $63 \%$ of the cases in MPS, HD and MI, respectively (Table 3 ). The overall diagnostic accuracy was $89 \%(81+17+21 / 133)$.

Seven cases of MI ( $26 \%$ ) were not associated with CF; five of them were premature. While, of the other twenty, related to $\mathrm{CF}$, only two were premature.

\section{Discussion}

Clinical manifestations of meconium plug syndrome (MPS), meconium ileus (MI) and neonatal Hirschsprung's disease (HD) are non-specific and consequently the radiologist plays a major role in discriminating among these disorders. In previous studies, the plain film findings of MPS were compared to MI [4] and to HD [8]; although, the number of patients and the parameters studied, were small. To our knowledge, the three disorders were never compared at the same time before.

Small left colon syndrome (SLCS) [9] was not considered as a separate entity. In our opinion, both MPS and SLCS are different terms referring to the same disorder. In
MPS, which is mostly observed in premature babies [4], the stressful condition has existed since birth, while in SLCS, a frequent finding in babies of diabetic mothers [9], there is intra-uterine stress, which probably results in an unused left colon. Others have also recognized MPS and SLCS as manifestations of the same underlying disease $[10,11]$. Prematurity in five cases of MI not associated to $\mathrm{CF}$, seemed to cause a MPS-like disease of small bowel, resulting from an overlap between MPS and MI [10]. Nevertheless such cases of MI fulfiled all diagnostic criteria for this disorder and were radiologically undistinguishable from the cases of MI related to CF. So MI is not always caused by $\mathrm{CF}[2,3]$.

The outcomes of radiographs (Table 1) were complex to differentiate among the three groups. Hence, discriminant analysis was used, which resulted in four main discriminating parameters, simplifying the scoring system (Table 2) for practical use.

Within the group of MPS, none of the cases, was predicted as HD (Table 3). This was important because MPS often mimics HD in the neonate [4,5].

Within the group of $\mathrm{MI}$, a high number of cases were mis-predicted. In our opinion, varying loop calibre in MI was essential (Table 1,2); in the absence of it, MI could easily be misdiagnosed as MPS or HD. In addition, when MI is suspected, ilial atresia should be kept in mind, although fluid levels in ilial atresia are said to be numerous coexisting with inverted U-shaped small bowel loops [12].

Within the group of $\mathrm{HD}$, the high diagnostic accuracy (Table 3) was meaningful for two reasons. Firstly it is vital to diagnose $\mathrm{HD}$ as soon as possible, for complications are serious [12]; secondly the most appropriate contrast medium could be selected to confirm the diagnosis.

The parameters which are the basis of the scoring system, will be discussed separately.

\section{Discriminating parameters}

Dilatation of bowel loops in HD, was less frequent when compared to MPS or MI (Table 1). Obstruction in HD, mostly located in the recto-sigmoid [11] and consequently having a large reservoir proximally, caused less dilatation.

Varying loop calibre, almost exclusively found in MI, was previously noted in $75 \%$ of the cases [13]. This 

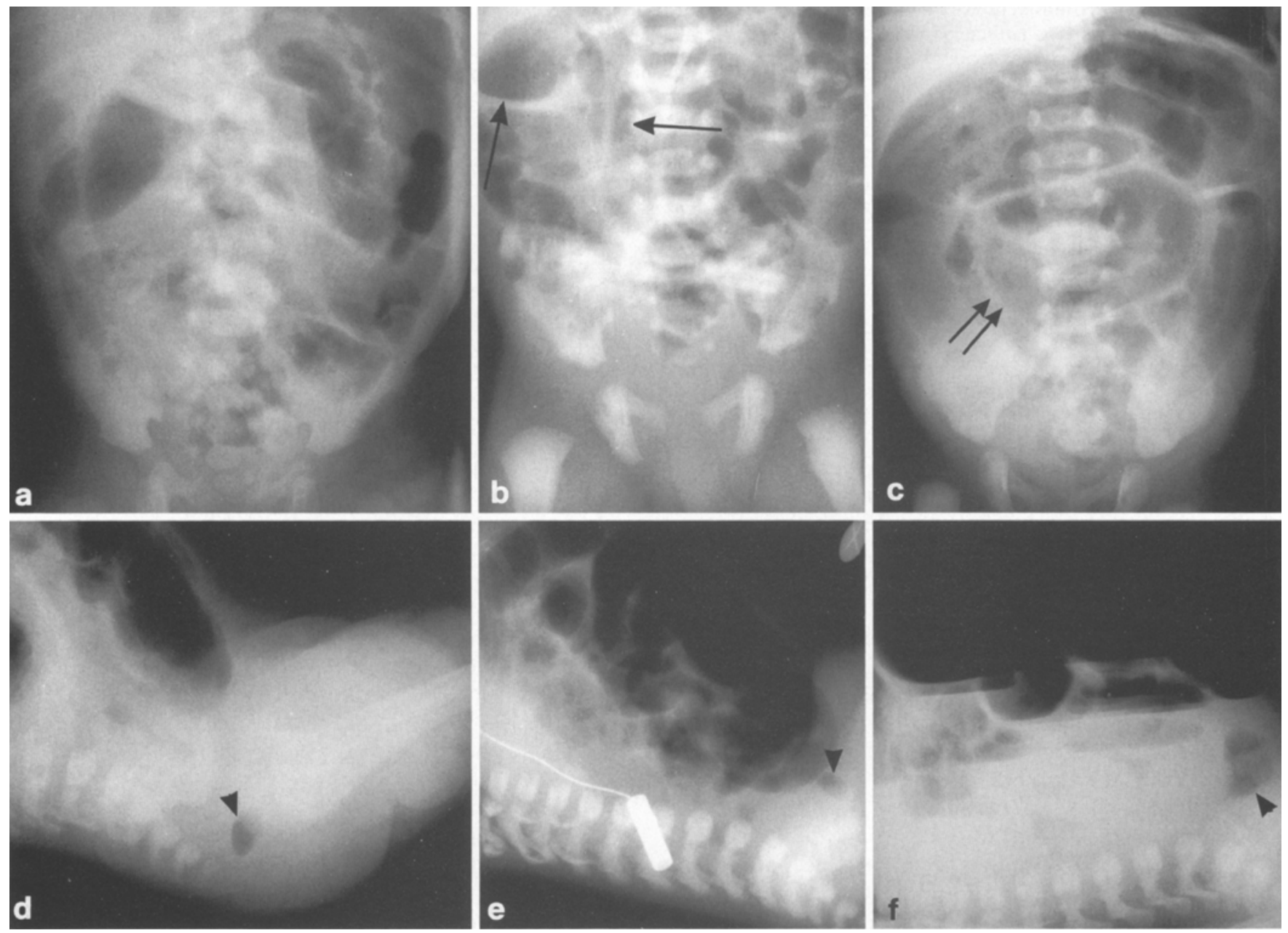

Fig.1 a-f. Three typical cases with low bowel obstruction, are illustrated by a supine radiograph and a supine lateral view with horizontal beam. a Meconium plug syndrome, b Meconium ileus and c neonatal Hirschsprung's disease. Dilated loops are visible in all three disorders, however meconium plug syndrome shows stretched loops with a moderate dilatation (a); the variation in loop calibre is only present in meconium ileus (arrows on $\mathbf{b}$ ); note that the scarce rectal gas can be seen in all three disorders (d-f, arrow heads). Bowel wall edema is well illustrated on (double arrows) and multiple fluid levels, characteristic of Hirschsprung's disease, on $\mathbf{f}$ parameter probably results from increased bowel peristalsis.

Fluid levels in MPS, absent in our material, were claimed to be present in $33 \%$ (3/9) to $42 \%$ (5/12) of cases $[4,14]$. However, close study of the infants [14], revealed that at the time of the diagnosis the obstruction with fluid levels, had existed much longer (2-6 days) as compared to that without fluid levels (1-2 days). In addition, recently none of seventeen cases of MPS showed fluid levels [10].

Fluid levels in MI are reported usually to be absent, due to the high viscosity of the meconium $[1,8]$. Their presence indicates complicated MI [7]. Others have observed fluid levels in complicated as well as in uncomplicated MI [6]. In our material only three out of seven cases of MI with fluid levels appeared to be complicated. Obviously the presence of this sign in MI was not necessarily a contra-indication for treatment with contrast enema, as proposed before [7].

Fluid levels in HD, previously observed in $90 \%$ of HD [4], compared to $71 \%$ in our study, results from stasis of fluid due to a diminished motility of the bowel, probably in combination with enterocolitis.

Colonic gas, particularly in combination with a small rectum, was highly suspicious of HD [12]. In our study, such a combination, though frequently seen in $\mathrm{HD}$, did also occur in MPS.

\section{Non-discriminating parameters}

Bowel wall edema i.e. constantly thickened and blunted mucosal folds in atonic bowel loops (Fig. 1), suggested enterocolitis, especially in combination with fluid levels [15]. Stretching of loops, resulting from swallowing air into an obstructed gut, would, in our opinion, signify only intact bowel function. Meconium mottling, regarded as a classic sign of MI $[1,8]$, was not solely present in MI. Similar results have been reported by others [6,7]. Rectal gas, and especially a small rectum, was often associated with HD $[5,12]$. Scarce or totally absent rectal gas, was invariably seen in all groups and had no diagnostic value (Table 1 , 
Table 2. For three score-functions, the weight (in points) of the parameters is given. The given points are obtained if a sign is present, otherwise the score is zero points. To each score a constant number of points (signified as "constant") is added. To classify patients, the three group-scores have to be calculated: the largest of the three indicates the most likely disorder. $\mathrm{DBL}=$ dilatation of bowel loops, $\mathrm{VLC}=$ varying loop calibre, $\mathrm{FL}=$ fluid levels and $\mathrm{CG}=$ colonic gas

\begin{tabular}{lcrrll}
\hline & DBL & VLC & FL & CG & constant \\
\hline MPS-score & 12 & 0 & 0 & 1 & 3 \\
MI-score & 11 & 12 & 3 & 0 & 0 \\
HD-score & 6 & 0 & 10 & 5 & 1 \\
\hline
\end{tabular}

Table 3. The actual group versus the predicted group according to score for cases of meconium plug syndrome (MPS), meconium ileus (MI) and neonatal Hirschsprung's disease (HD). For each case three scores were calculated by using the scoring system presented in Table 2, thereby indicating the most likely disorder

\begin{tabular}{|c|c|c|c|c|c|c|c|}
\hline \multirow{3}{*}{$\begin{array}{l}\text { Predicted } \\
\text { groups }\end{array}$} & \multicolumn{7}{|c|}{ Actual groups } \\
\hline & \multicolumn{2}{|c|}{$\begin{array}{l}\text { Meconium } \\
\text { plug syndrome }\end{array}$} & \multicolumn{2}{|c|}{$\begin{array}{l}\text { Meconium } \\
\text { ileus }\end{array}$} & \multicolumn{3}{|c|}{$\begin{array}{l}\text { Hirschsprung's } \\
\text { disease }\end{array}$} \\
\hline & $(n)$ & {$[\%]$} & $\overline{(n)}$ & {$[\%]$} & $(n)$ & {$[\%]$} & \\
\hline$\overline{\mathrm{MPS}}$ & 81 & 99 & 7 & 26 & 3 & 12 & 91 \\
\hline MI & 1 & 1 & 17 & 63 & 0 & - & 18 \\
\hline HD & 0 & - & 3 & 11 & 21 & 88 & 24 \\
\hline total & 82 & & 27 & & 24 & & 133 \\
\hline
\end{tabular}

Fig.1). Perforation was not seen in MI. In fact, there were four perforated cases of MI: two of them occured during contrast enema and two were recognized during operation. Such perforations were probably small or sealed off but one must remain cautious during contrast enema treatment [16].

In conclusion, in most cases of meconium plug syndrome, meconium ileus and neonatal Hirschsprung's disease, an accurate plain film diagnosis can be predicted by using a scoring system. Such a diagnosis can be regarded as a sound basis for further diagnostic or therapeutic procedures.

\section{References}

1. Holsclaw DS, Eckstein HB, Nixon HH (1965) Meconium ileus. A 20-year review of 109 cases. Am J Dis Child 109: 101

2. Dolan TF Jr, Touloukian RJ (1974) Familial meconium ileus not associated with cystic fibrosis. J Pediatr Surg 9: 821

3. Shigemoto H, Endo S, Isomoto T, Sano K, Taguchi K (1978) Neonatal meconium obstruction in the ileum without mucoviscoidosis. J Pediatr Surg 13:475

4. Pochaczevsky R, Leonidas JC (1974) The meconium plug syndrome. Roentgen evaluation and differentiation from Hirschsprung's disease and other pathologic states. AJR Radium Ther Nucl Med 120: 342

5. Schey WL, White H (1971) Hirschsprung's disease. Problems in the roentgen interpretation. AJR Radium Ther Nucl Med 112: 105

6. Leonidas JC, Berdon WE, Baker DH, Santulli TV (1970) Meconium ileus and its complications. A reappraisal of plain film roentgen diagnostic criteria. AJR Radium Ther Nucl Med 108:598

7. Caffey J (1985) Pediatric X-ray diagnosis, 8th edn. Year Book Medical Publishers, Chicago, pp 1838-1858

8. Tucker AS, Izant RJ Jr (1971) Problems with meconium. AJR Radium Ther Nucl Med 112:135

9. Davis WS, Allen RP, Favara BE, Slovis TL (1974) Neonatal small left colon syndrome. AJR Radium Ther Nucl Med 120:322

10. LeQuesne GW, Reilly BJ (1975) Functional immaturity of the large bowel in the newborn infant. Radiol Clin North Am 13:33

11. Berdon WE, Slovis TL, Campbell JB, Baker DH, Haller JO (1977) Neonatal small left colon syndrome: its relationship to aganglionosis and meconium plug syndrome. Radiology 125: 457

12. Swischuk LE (1989) Radiology of the newborn, infant, and young child. 3rd edn. William and Wilkins, pp 436446 and pp 474-487

13. Eklöf $O(1977)$ Current concepts in pediatric radiology. Springer, Berlin Heidelberg New York, pp 54-58

14. Clatworthy HW Jr, Howard WHR, Lloyd J (1956) The meconium plug syndrome. Surgery 39: 131

15. Bill AH, Chapman ND (1962) The enterocolitis of Hirschsprung's disease. Its natural history and treatment. Am J Surg 103: 70

16. Caniano DA, Beaver BL (1987) Meconium ileus: a fifteen-year experience with forty-two neonates. Surgery 102: 699

Prof. Dr. M. Meradji

Department of Pediatric Radiology

Sophia Children's Hospital

Gordelweg 160

NL-3038 GE Rotterdam 